\title{
ESTIMATION OF UNKNOWN PARAMETERS IN SYSTEM DYNAMICS MODELS USING THE METHOD OF SIMULATED MOMENTS
}

\author{
Hazhir Rahmandad \\ Mohammad S. Jalali \\ Industrial and Systems Engineering \\ Virginia Tech \\ Falls Church, VA 22043, USA
}

\author{
Hamed Ghoddusi \\ Engineering Systems Division \\ Massachusetts Institute of Technology \\ Cambridge, MA 02139, USA
}

\begin{abstract}
In principle the Method of Simulated Moments (MSM) combines simulation-based methods (e.g. Monte Carlo methods) with non-parametric statistical estimations techniques such as General Method of Moments (GMM). The MSM is useful when there are empirical data related to the behavior of different entities. Different statistical moments (e.g. mean, variance, correlation, etc.) of empirical data can be matched against the moments of model-generated data in order to estimate some structural parameters of the model. In this paper, we introduce the MSM as a non-parametric method of estimating the parameters of dynamic models. The major value of the MSM for estimating dynamic models is in its flexibility to be used with any type of data, including cross-sectional data and time series data.
\end{abstract}

\section{INTRODUCTION}

Increasingly, dynamic modelers face problems where estimating model parameters from numerical empirical data is a requirement. This trend is partly motivated by increasing availability of numerical data from a large number of ongoing and one off data collection projects that survey different concepts of interest to dynamic modelers, from individuals and firms to disease incidences and measures of economic performance, just to name a few. For example, as of March 2013, Data.gov portal contains data on over 370 thousand machine readable datasets. Another driver of this trend is the increasing application of dynamic models, beyond case specific corporate projects, to theoretical and academic problems (Repenning 2003, Sterman 2006). In these cases generic models for a category of objects (e.g. individuals, firms, and countries) are desired. Parameterizing such models requires specifying the different parameters that quantify similarities and differences across different objects, a goal often dependent on using robust and replicable parameter estimation procedures. In fact, in light of rapid growth and dissemination of improved parameter estimation methods for model calibration, hypothesis testing, and policy recommendation in social and behavioral disciplines, continued relevance of any modeling sub-discipline may partially be tied to its ability to remain up-to-date with the best available tools in this domain.

Closely tied to the advances of digital computer revolution, at its inception the field of system dynamics (SD) was ahead of many approaches available in social sciences in using advanced analytical methods of the time (Forrester 1961), and kept this edge for many years. For example advanced filtering and estimation methods were introduced into the SD literature in the 70's (Peterson 1975). However, over the last three decades the research in SD has largely focused on diverse applications of the original toolbox, with limited methodological expansions in parameter estimation domain. In contrast research in econometrics and other related fields have provided many relevant tools over this period (Greene 2012). As a result currently many studies in SD do not report formal parameter estimates common in social science research, or when calibration is pursued, typical measures of confidence in estimated parameter values are not reported. While formal parameter estimation may not be feasible for many modeling problems, expert dynamic modelers should be equipped with the relevant tools when numerical data is available, model purpose re- 


\section{Rahmandad, Jalali, and Ghoddusi}

quires reliable parameter estimates, or the audience requires formally estimated parameters and confidence intervals.

Hand-calibration is commonly practiced for assigning parameter values (Lyneis and Pugh 1996). When formal parameter estimation procedures are used, modelers typically compare time series data against the same variables in a model, and minimize the weighted sum of a function of the error term by changing the uncertain parameters until best fitting estimates are found through a nonlinear optimization algorithm (Oliva 2003). The error function is frequently defined as the squared error but absolute error and absolute percent error terms are also common (Sterman 2000). Weights for different data points are usually given based on the confidence the researcher has in the accuracy of the data and its relevance to the problem at hand. When reported, confidence intervals are calculated using normality and independence assumption for error terms which, with weights proportional to the reciprocal of error variance, would turn least squared error estimates into maximum-likelihood estimates (MLE). Bootstrapping methods are also sometimes used for estimating confidence intervals (Dogan 2007).

While these approaches cover many important estimation challenges, they each include some shortcomings. Ad hoc selection of the error term and the weights for different data points reduces the consistency of the methods and their ability to provide confidence intervals. Normality and independence may regularly be violated which negate the benefits of MLE when using squared errors. Bootstrapping, while flexible, increases the computational costs significantly and as a result may prove infeasible for many realistically-sized problems. Finally, all these methods rely on having time series data, and cannot extract from distributions in cross-sectional data the dynamics that have led to those distributions. In general the following characteristics signify the estimation procedures ideal for dynamic modelers:

- Model Independence: given that most dynamic models do not follow a fixed structural form (e.g. linearity), estimation procedures that are independent of model structure are most beneficial.

- Analytical confidence intervals: Ability to find confidence intervals analytically is important because of the computational costs of optimizing non-linear dynamic models and replications needed for bootstrapping methods.

- Assumption-free error terms: Independence and distributional assumptions on error terms for dynamic models are not always easy to justify so methods with fewer such assumptions are preferred.

- Applicability to diverse data types, including both time series and cross-sectional data. No single method fully satisfies all these requirements, therefore modelers need to choose from a menu of available estimation methods to match their problem requirements.

In this paper we offer an introduction to the Method of Simulated Moments (MSM) for application to dynamic modeling problems that represents the characteristics discussed above. While the MSM has become a major econometrics tool for the past two decades, it has been rarely applied in the system dynamics literature. Barlas (2006), in the design of Behavior Pattern Testing (BTS II) approach and software, uses some of the basic ideas of the MSM, to match moments of model against data, but does not draw on the MSM literature or discuss issues related to confidence levels. Rahmandad and Sabounchi (2011) adapt the MSM to estimating the parameters of an individual weight gain and loss model. While this application follows the basic ideas of the MSM, it has some differences from the canonical the MSM procedure which will be discussed in section 4 .

The basic idea of the MSM is to define appropriate moments of data and, by changing uncertain parameters, minimize the difference between those moments and their simulated counterpart coming from the model. Moments could be any function of data points available. However for analytical confidence intervals to be available, one needs these moments to be normally distributed, often meaning that each moment is an average across a function of multiple independent observations coming from the same underlying distribution (then normality follows from the central limit theorem). In practice those observations (that feed into the moments calculations) are picked either from time series data when a system is in steady state (e.g. stock prices over time), or at similar points in the life of similar units of observations 


\section{Rahmandad, Jalali, and Ghoddusi}

(e.g. all 5-year old individuals in a country). Typically randomness plays an important role in how these units have ended up with different observed values (e.g. different weights for similarly-aged individuals). As a result, the MSM is best fitted for dynamic modeling problems when some of the following problem characteristics are present:

- Population data. The MSM is suitable for estimation of generic models to population data. Different units of data such as individuals, firms, and countries could be available. For each unit one or more data items (e.g. weight, height, and age for individual data) could be available.

- Role of random processes. The MSM could be a good choice when models include stochastic processes that drive the model, and their impact on the model behavior is reflected in the data against which the model is to be calibrated, e.g. when we are trying to match the variance observed across multiple units.

- Cross-sectional data. The MSM applies to both cross-sectional and time series data. Where-as time series data include multiple data points for the same unit over time, cross-sectional data includes data points for multiple units at the same time. The MSM may be the only viable choice for estimating dynamic models when data is cross-sectional as it allows us to extract the information about the historical trajectories of units hidden in their cross-sectional distributions.

- Confidence Intervals. The MSM would be a suitable choice when analytical confidence intervals are sought.

\section{HISTORICAL BACKGROUND}

MSM is an offspring of the Method of Moments (MM). Here we provide a quick review of the MM.

\subsection{Method of Moments}

As a classical estimation method in statistics, the MM is based upon finding unknown parameters of a certain distribution by relating these parameters to the moments of the distribution and then using empirical moments (obtained from data) to back up the unknown distribution parameters. The unknown parameters are estimated by equating those empirical moments with unobservable population moments and then solving those theoretical equations (Raychaudhuri 2008). We explain this using a few examples.

\subsubsection{Example 1: Normal Distribution}

The most convenient (and straightforward) example for MM estimation can be expressed using a case for the Normal distribution. Suppose you have collected a large sample of independent and identically distributed (i.i.d) observations for an experiment (a sample of heights of individuals in a country). We are confident that the true (or the best fitting) functional form for the distribution is Normal. However, we do not know the values for the mean $(\mu)$ and variance $\left(\sigma^{2}\right)$ of this distribution to fully characterize it.

Estimating the mean is easy since we can rely on the Law of Large Numbers (L.L.N) which suggests that the mean of a large sample of trials will converge to its true mean. So we can simply calculate $\mu_{s}=\frac{\sum_{i=1}^{n} e_{i}}{n}$ and use it as the best estimator of $\mu$. Now we need to estimate the variance. Remember the formula for variance $\sigma^{2}=\mathrm{E}\left(X^{2}\right)-(\mathrm{E}(X))^{2}$. Here we know $\mathrm{E}(X)$ from our estimation of mean. Moreover, we can calculate $\mathrm{E}\left(X^{2}\right)$ using our sample $\mathrm{E}\left(X^{2}\right) \approx \frac{\sum_{i=1}^{n}\left(e_{i}^{2}\right)}{n}$. Plugging back these two values into the variance equation we obtain $\sigma^{2}=\frac{\sum_{i=1}^{n}\left(e_{i}^{2}\right)}{n}-\left(\frac{\sum_{i=1}^{n} e_{i}}{n}\right)^{2}$. Note that our ability to estimate 


\section{Rahmandad, Jalali, and Ghoddusi}

the two parameters here was dependent on knowing the analytical formulas that specify the unknown parameters $\left(\mu, \sigma^{2}\right)$ as a function of quantities that can be directly measured.

\subsubsection{Example 2: Binomial Model}

Now suppose that our data points are drawn from a binomial distribution $\operatorname{Bi}(p, n)$ in which $p$ is the probability of success and $n$ is the number of trials. Let us assume we have several observations from this distribution, but do not know the value of parameters $p$ and $n$. We know that the mean and variance of a binomial distribution is given by $\mu=n p$ and $\sigma^{2}=n p(1-p)$. Similar to the previous example we can calculate the first and second moments of the data $\left(\mathrm{E}(X)\right.$ and $\left.\mathrm{E}\left(X^{2}\right)\right)$. Using the formula for the variance $\left(\sigma^{2}=\mathrm{E}\left(X^{2}\right)-(\mathrm{E}(X))^{2}\right)$ we express the second moment of data using the parameters of the binomial model, $\mathrm{E}\left(X^{2}\right)=n p(1-p)+(n p)^{2}$. Therefore we can now use the first and second moment equations together to provide a system of two equations and two unknowns which can be solved to recover $(n, p)$. Specifically, $p=1-\frac{\mathrm{E}\left(X^{2}\right)-\mathrm{E}(X)^{2}}{\mathrm{E}(X)}$ and $n=\frac{\mathrm{E}(X)}{p}$.

These two examples provide some intuition over the merits and difficulties of MM techniques. While for certain probability distributions MM can be used to recover parameter values through analytical expressions, it faces two major challenges. First, we need to know the true functional form of the distribution of outcomes. Second, we should be able to express the parameters of the distribution in terms of the data moments, a task only feasible for a small set of probability distributions. For many distributions we cannot find an analytical (close-form) solution to relate moments to parameters. Structural models in general and systems dynamics models in particular usually do not have an analytical solution to relate the output of the model to its structural parameters. Therefore, the classical method of moments discussed above is not directly applicable to these models.

\section{FROM METHOD OF MOMENTS TO METHOD OF SIMULATED MOMENTS}

Mcfadden (1989) was the first who proposed using simulation instead of trying to solve the moment conditions analytically. His paper was focused on discrete-response models (multinomial Probit), however he provided theoretical foundations for more general models. Mcfadden (1989) believed that an unbiased simulator is used in the MSM where the simulation errors are independent across observations, and the variance introduced by simulation will be controlled by the law of large numbers operating across observations. Lee and Ingram (1991) and Duffie and Singleton (1993) extended the framework and provided a rigorous treatment of the MSM estimators for time-series and panel-data cases and provided relevant statistics for making tests. Duffie and Singleton (1993) showed that the MSM estimator is, under regularity conditions, consistent and asymptotically normal. Since then the MSM has been widely used in various sub-fields of economics such as finance (both asset-pricing and corporate finance), macroeconomics, Industrial Organization (IO), international trade and labor economics.

\section{AN EXAMPLE FROM THE DYNAMIC MODELING LITERATURE}

As mentioned earlier, Rahmandad and Sabounchi (2011) adapt the MSM to estimating the parameters of an individual weight gain and loss model. In this section we provide a brief overview of this application to provide a more concrete example of the MSM use. A simple model of individual's body mass, consisting of fat mass and fat free mass, is developed. The model included a few uncertain parameters. In absence of time series data, those parameters were estimated from cross sectional data on individual weights coming from the National Health and Nutrition Examination Survey (NHANES). NHANES 2005-2006 population of 5,971 subjects was categorized into 110 subpopulations based on different ethnicities (5 


\section{Rahmandad, Jalali, and Ghoddusi}

ethnicities), genders ( 2 genders) and age (11 age groups). For each population group two moments, average body weight and variance of body weight, were calculated as the moments to be matched, leading to a total of 220 moments to match.

On the other hand, the model was replicated (using subscripts in Vensim software) for 5,971 instances that matched the demographic characteristics (Age, Gender, Ethnicity) of the NHANES sample in year 2006. Initial body weight and fat fraction for these individuals were drawn from distributions of another NHANES sample in 1999-2000. Note that each round of NHANES uses a sample different from other rounds, thus we cannot track the same individual over time and the data is cross-sectional. The model was then simulated to grow this synthetic population from their initial age in year 2000 to their final age (consistent with NHANES sample) in year 2006. Mean and variance of weight for different subpopulations in the simulated population was calculated in year 2006, and compared against the 220 moments coming from the data. Weighted sum of squared errors was calculated using weights of reciprocal of variance in each moment, itself calculated using variance and kurtosis of different moments. This error was minimized by changing 17 uncertain parameters using the Vensim internal optimization engine. The estimated parameters provided the minimum error. As a result, the authors were able to estimate a dynamic model, including individual growth mechanisms, from cross sectional data with individuals in different age groups.

While this application follows the basic ideas of the MSM, it has some differences from the canonical the MSM procedure. First, in this application the number of moments (220) is larger than many typical applications, in which the numbers of moments and parameters to be estimated are in the same order of magnitude. Second, given the computational costs in this setting, each moment was only simulated once whereas typically multiple simulations, using different noise seeds, shall provide the estimation for the moment, before it is compared with data. Finally, confidence intervals were not reported in this application.

\section{BASICS OF THE MSM}

Let's go back to the previous example. Suppose you have built a model which captures the dynamics of people's body weight as a function of their initial weight, eating and physical exercise habits, genetics, age, gender and other fixed and time-varying characteristics. People differ both in terms of their idiosyncratic characteristics (genetics, initial weight, etc.) and their environmental factors (e.g. quality of food, cost to exercise, social eating habits, etc.). By changing initial conditions and model parameters one will get different dynamic paths for the agent's (individual's) weight as a function of her age. Suppose we have data on the weight of several children of age 10 (our initial value) as well as at ages 11 and 12 . Further assume that we are interested in estimating a structural parameter (e.g. average weight growth per year) which determines the weight path as a function of initial weight.

By fixing this (unknown) parameter to an initial value and simulating the model with all empirical values for the initial weight (age 10), we will generate different paths of weight-age for a simulated population the same size as the number of subjects in our dataset. Now we can compare the distribution of model-predicted weight profiles at ages 11 and 12 against the empirical distributions. Specifically we can compare the mean and variance of weight for simulated population at ages 11 and 12 against the mean and variance at the same ages observed in the data. It is likely that our initial choice for the structural parameter leads to mean and variance weights different from those observed in the data. However these simulated moments are a function of the parameter. By changing the structural parameter of the model we will change both the mean and the variance of simulated weight values. We can therefore use an optimization method to search for the parameter value that minimizes the difference between model generated mean and variance and their empirical values over all available moments (i.e. mean and variance of age at ages 11 and 12). This is the core idea behind the method of simulated moments: we simulate the moments of the model to find simulated counterparts for observed data, then change the structural parameters until the simulated moments match the observations as closely as possible. 


\section{Rahmandad, Jalali, and Ghoddusi}

\section{FORMAL DEFINITIONS}

Consider a fully-specified model, i.e. a model that can be simulated given a set of parameter values. Assume that there are $d$ unknown parameters which we are interested in estimating. Let's assume that our empirical data $\left\{x_{t}\right\}$ are observed for $T$ different agents. There are $P$ moments functions (sometimes called descriptive statistics) that are available in the data for each agent and $m_{i}(t), i \in\{1, \ldots, P\}$ is the $i$ th moment of the data for agent t. The $i^{\text {th }}$ element of vector of data moments $\tilde{M}_{i}$ is defined by:

$$
\tilde{M}_{i}=\frac{1}{T} \sum_{t=1}^{t=T} m_{i}(t)
$$

Notice that since we only have access to a sample of data for estimating moments, the true moments of population from which the data sample is collected are approximated by empirical moments $\widetilde{M}_{D}$. The true functional form of the system's dynamics which lead to output $g($.$) is approximated by the model's$ output $\hat{g}($.). The output of the model is a function of known parameters vector $Z$, unknown parameters vector $\theta$ (to be estimated) and random inputs $u$. Choosing different values for $u$ will generate different values for $g$. We assume that the model is correctly specified so that $\hat{g}($.$) is an unbiased estimator of$

$$
g(.): \mathrm{E}(\hat{g}(Z, \theta, u))=g(Z, \theta)
$$

This ensures that if we generate a large enough sample of outputs using a true random stream of inputs $u_{t}$ the arithmetic average of the model output should generate a reasonable approximation of the real-world processes that generate the observations.

$$
m_{i}(\hat{g}(.)) \approx \frac{1}{N} \sum_{j=1}^{N} \frac{1}{T} \sum_{t=1}^{T} m_{i}\left(\hat{g}\left(Z, \theta, u_{t}\right)\right)
$$

Equation above can be understood as following. The component $\sum_{t=1}^{T} m_{i}\left(\hat{g}\left(Z, \theta, u_{t}\right)\right)$ represents the fact that moments may need a number of observations to be calculated. For example, think of variance as a popular moment. The standard formula of variance $\mathrm{E}(X-\mathrm{E}(X))^{2}$ suggests that we should calculate the empirical average of $\left(X_{t}-\mathrm{E}(X)\right)^{2}$ for all observations $t \in 1, \ldots, T$. As a convention the notation of $\sum_{t=1}^{T} m_{i}\left(\hat{g}\left(Z, \theta, u_{t}\right)\right)$ represents all types of averaging operations which are required for calculating different moments.

It is necessary to make sure that the moments we estimate from the model are not affected by sampling bias. If the size of a model-generated sample is not too large (i.e. $T$ is small) we will get different estimates of moments per each sample. Repeating the sampling for a large number of times $(N)$ and averaging each of the moments over these samples reduces this bias.

A necessary (but not sufficient) condition for being able to identify the model is to have more moment conditions than unknown parameters. Otherwise, we will have free parameters and the unknowns will not be determined uniquely. Ideally, we require more moment conditions than the unknown parameters $(P>>d)$. We call this over-identification.

The previous description was focused on a single set of agents. If we want to simulate $K$ collective entities (communities, cities, classrooms) in parallel (i.e. generate a panel of observations) we will generate a larger number of observations. In this case the model generated moments would be expressed as:

$$
m_{i}(\hat{g}(.)) \approx \frac{1}{N K} \sum_{j=1}^{N k} \frac{1}{T} \sum_{t=1}^{T} m_{i}\left(\hat{g}\left(Z, \theta, u_{t}\right)\right)
$$




\section{Rahmandad, Jalali, and Ghoddusi}

The core of the MSM is to minimize the (weighted) difference between the empirical and simulated moments by changing the unknown parameters. The estimated parameter set is the value of parameters that minimizes this difference. Specifically, with vector of simulated moments $M_{S}$ consisting of $m_{i}(\hat{g}()$.$) elements and the <P \times P>$ matrix $W$ for weighting the moment conditions:

$$
\theta^{*}=\operatorname{argmin}\left(M_{S}-M_{D}\right) W\left(M_{S}-M_{D}\right)
$$

If an estimate moment is very sensitive to the random input path, it will generate possibly diverse values across different rounds. On the other hand, those moments that are more robust against the choice of the sample will show smaller dispersion. Using the inverse of variance for matrix $W$ helps us give more weight to more robust moments and reduce the importance of those that change a lot from one round to another.

\section{CHOOSE THE MOMENT CONDITIONS}

Usually the first and second moments of model's outcomes (mean and variance) are good candidates to use. Remember that the number of moment conditions should be (equal to or) larger than the number of unknown parameters. Thus, depending on the number of parameters you should decide to use informative moment conditions. The most informative moments are the ones that 1) are sensitive to at least one of the unknown parameters (discussed further under Conditions for Identification), 2) have rather small variances. The larger the variance of a moment (across multiple simulations) the less informative it is for estimation.

In addition to single variable moments (e.g. mean and variance of one variable) one can also try cross-variable moments such as the correlation/covariance between two output variables, autocorrelation of a variable with itself, etc.

Adding an additional parameter to be estimated is costly, both in terms of computation time and in terms of identification strategy. So, we suggest trying to estimate as many parameters as possible (if it is possible and reasonable) from other sources (e.g. review of literature, regressions, etc.) and leave the minimum number of parameters for the MSM technique.

\section{CONDITIONS FOR IDENTIFICATION}

The right choice of moment conditions is the most crucial step in identifying the model and recovering model parameters. Identification of a model using the MSM requires that the model-generated moment conditions should fit their empirical counterparts if and only if the structural parameters equal their true values. Otherwise, the model will generate spurious results. Furthermore, the sufficient condition for identification is a one-to-one mapping between the structural parameters and a subset of the moment restrictions of the same dimension. Because our models often do not yield such a closed-form mapping, to help ensure an identified model, one should choose moments that are sensitive to variations in the structural parameters. One way to check this is to run one dimensional sensitivity analyses on each parameter to be estimated, and check if selected moment conditions vary substantially with changes in parameter values. If a moment condition does not vary much or if its response to changes in parameters is not smooth and monotonic then using that moment may not be very informative and even may cause the optimization engine to stop in a local optimum or never converge to an optimal solution. Figure 1 shows examples of informative and non-informative moments.

The moment specified by the solid line moves smoothly as the unknown parameter changes and has a unique well-defined extreme point (minimum in figure 1). Therefore, minimizing the distance between this function and the empirical moment will generate a unique parameter value. On the other hand, the moment represented by the dashed-line is not informative. It is not very sensitive to changes in parameter value. We cannot even be sure that these small changes are due to true response of the model to various parameter values or are the artifact of computational or sampling errors (though if the graph is smooth 
these conjectures will be less valid). Moreover, the moment shows multiple extreme values and thus minimizing its distance from the empirical value will not identify a unique value.

\section{OPTIMIZATION ROUTINE AND ITERATION}

This is the most computationally-challenging step of the MSM procedure. We need to minimize the weighted distance of model-generated moments from empirical moments. More formally:

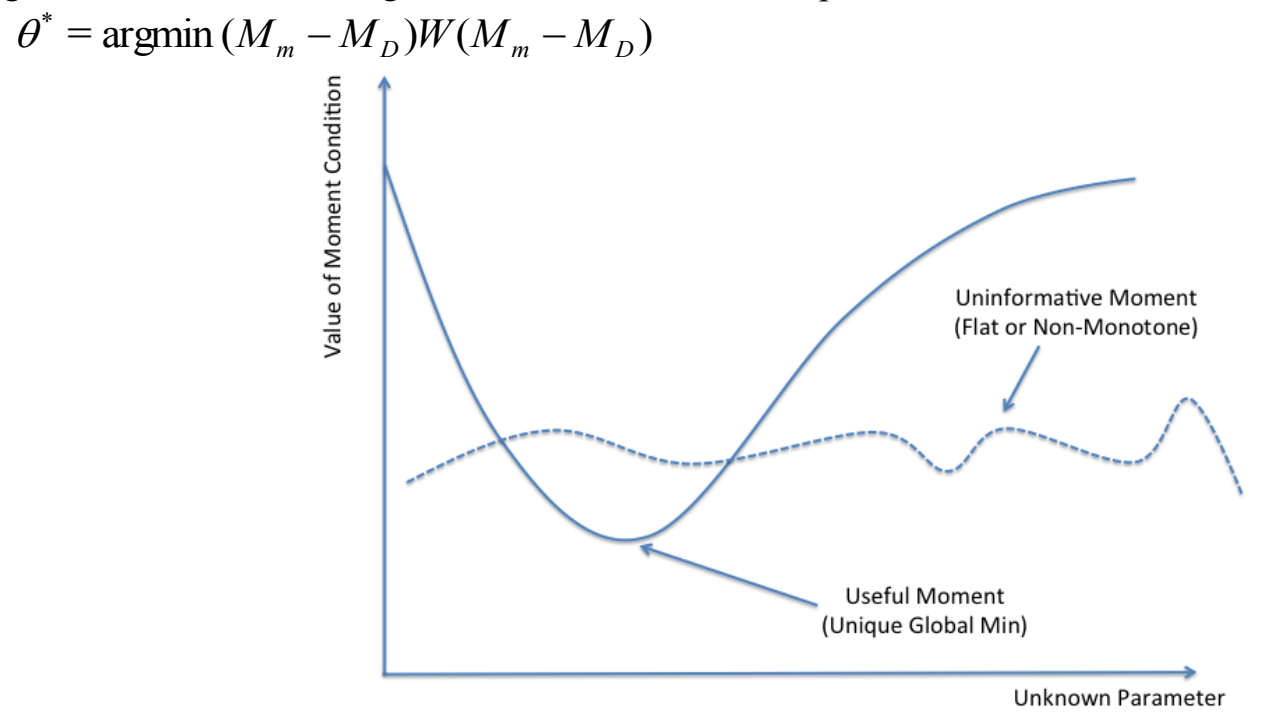

Figure 1: Informative and non-informative moments

We need to use numerical optimization routines to find the minimum of the total error function. A smart choice of initial values for parameters may facilitate the quicker convergence of the optimization routine significantly. Any numerical optimization method requires a tolerance rule to stop. This will be given as the error tolerance for the objective function $\left\|Q^{i}-Q^{i-1}\right\| \varepsilon_{Q}$ as well as for the parameters $\left\|\widetilde{\theta}^{i}-\widetilde{\theta}^{i-1}\right\| \varepsilon_{\theta}$. Similar to any non-linear optimization routine, the MSM estimator may fall into the trap of a local maximum. Moreover, if some of moment conditions are not very informative, they would have low sensitivity to parameter values the problem may face a flat value function which makes it very difficult to progress and converge. To avoid introducing sampling error into rounds of simulation we should work with the same random sample of shocks in each period to make sure that changes in results are due to changes in structural parameters and not the random sample.

\section{OVER-IDENTIFICATION TEST}

When the model is over-identified some of moment conditions will be different zero. In this case a subset of moments, whose number is equal to the number of unknowns, will satisfy zero and the remaining (p-q) moment conditions can be different than zero. For a good model, whose structure and parameter values are close to the true system, the value of these conditions should not be substantially different from zero. One can use the standard $\mathrm{J}$ test for testing over-identification. The J-statistic is given by

$$
J=\frac{N T}{1+N}\left(\left(M_{m}-M_{D}\right) W\left(M_{m}-M_{D}\right)\right): \chi^{2}(p-q)
$$

\section{ROBUSTNESS CHECKS}

The MSM uses numerical methods to find the minimum of the objective function. Therefore, the results might be sensitive to initial values, the precision of the search algorithm (the level of error tolerance), and 


\section{Rahmandad, Jalali, and Ghoddusi}

the quality of algorithm to distinguish local and global extreme points. We recommend to re-run optimization using distant initial values to check if the results are sensitive to the choice of initial value.

\section{CRITIQUES AND SHORTCOMINGS OF THE MSM}

In principle the MSM shares some of the concern of being 'black box' regarding the transparency of dynamic systems modeling. Some researchers believe that it provides a large degrees freedom for choosing the moment conditions which serve the interests of the modeler. By imposing different moment conditions one may recover different structural parameters and as long as there are enough meaningful moment conditions the researcher has the luxury of using an arbitrary subset of moments. This critic compares the MSM method to standard econometrics techniques which are more easily available for outsiders and provide a better chance of independent verification of the estimation results.

Adda and Cooper (2003) believed that for implementing the MSM, a large number of simulations is needed to compute the standard errors of the estimator. Using Monte-Carlo analysis, Ruge-Murcia (2012) presented that even when simulated series are short, the MSM works and it can deliver accurate estimates; however, the distribution of the estimates is not efficiently approximated by the asymptotic normal distribution.

There are ways to address some of these concerns. Providing graphs showing the sensitivity of the likelihood function to parameter values is one way to convince the others that the moments were indeed informative. Providing the data (if they are not propriety) and the programming codes enables others to check the results independently.

\section{CONCLUSION}

Over the last three decades the research in system dynamics has largely focused on diverse applications of the original toolbox, with limited methodological expansions in parameter estimation domain. While formal parameter estimation may not be feasible for many modeling problems, expert dynamic modelers should be equipped with the relevant tools when numerical data is available, model purpose requires reliable parameter estimates, or the audience requires formally estimated parameters. In this paper we offer an introduction to the MSM for application to dynamic modeling problems. The basic idea of this method is to define appropriate moments of data and, by changing uncertain parameters, minimize the difference between those moments and their simulated counterpart coming from the model.

Given that most dynamic models do not follow a fixed structural form (e.g. linearity), estimation procedures such as the MSM that are independent of model structure are most beneficial. Moreover, independence and distributional assumptions on error terms for dynamic models are not always easy to justify, so the MSM with fewer such assumptions is preferred. The MSM is especially useful when error terms do not follow any well-established distribution. It could be also a good tool when models include stochastic processes that drive the model, and their impact on the model behavior is reflected in the data against which the model is to be calibrated, e.g. when we are trying to match the variance observed across multiple units.

The MSM is also applicable to diverse data types, including both time series and cross-sectional data. It may be the only viable choice for estimating dynamic models when data is cross-sectional as it allows us to extract the information about the historical trajectories of units hidden in their cross-sectional distributions.

\section{REFERENCES}

Adda, J., and R. W. Cooper. 2003. Dynamic economics: quantitative methods and applications. Cambridge, Mass.: MIT Press.

Barlas, Y. 2006. Model validity and testing in system dynamics: Two specific tools. Paper read at 24th International Conference of the System Dynamics Society, at Nijmegen, The Netherlands. 


\section{Rahmandad, Jalali, and Ghoddusi}

Dogan, G. 2007. "Bootstrapping for confidence interval estimation and hypothesis testing for parameters of system dynamics models." System Dynamics Review no. 23 (4):415-436.

Duffie, D., and K. J. Singleton. 1993. "Simulated Moments Estimation of Markov-Models of Asset Prices." Econometrica no. 61 (4):929-952.

Forrester, J. W. 1961. Industrial Dynamics. 1 ed. Cambridge: The M.I.T. Press.

Greene, W. H. 2012. Econometric analysis. 7th ed. Boston: Prentice Hall.

Lee, B.-S., and B. F. Ingram. 1991. "Simulation estimation of time-series models." Journal of Econometrics no. 47 (2-3):197-205. doi: 10.1016/0304-4076(91)90098-X.

Lyneis, J. M., and A. L. Pugh. 1996. Automated vs. 'Hand' Calibration of System Dynamics Models- An Experiment With a Sample Project Model. Paper read at the 14th International Conference of the System Dynamics Society, at Cambridge, Massachusetts.

Mcfadden, D. 1989. "A Method of Simulated Moments for Estimation of Discrete Response Models without Numerical-Integration." Econometrica no. 57 (5):995-1026.

Oliva, R. 2003. "Model calibration as a testing strategy for system dynamics models." European Journal of Operational Research no. 151 (3):552-568. doi: Doi 10.1016/S0377-2217(02)00622-7.

Peterson, D. W. 1975. Hypothesis, estimation, and validation of dynamic social models- Energy demand modeling, Massachusetts Institute of Technology, Cambridge, MA.

Rahmandad, H., and N. Sabounchi. 2011. Building and estimating a dynamic model of weight gain and loss for individuals and populations. In the 29th International Conference of the System Dynamics Society. Washington, DC.

Raychaudhuri, S. 2008. Introduction to monte carlo simulation. In Simulation Conference, 2008. WSC 2008. Winter.

Repenning, N. P. 2003. "Selling system dynamics to (other) social scientists." System Dynamics Review no. 19 (4):303-327. doi: Doi 10.1002/Sdr.278.

Ruge-Murcia, F. 2012. "Estimating nonlinear DSGE models by the simulated method of moments: With an application to business cycles." Journal of Economic Dynamics and Control no. 36 (6):914938. doi: 10.1016/j.jedc.2012.01.008.

Sterman, J. D. 2006. "Learning from evidence in a complex world." American Journal of Public Health no. 96 (3):505-514. doi: 10.2105/ajph.2005.066043.

Sterman, J. D. 2000. Business dynamics: systems thinking and modeling for a complex world. 1 ed. Boston: McGraw-Hill/Irwin.

United States Government. 2012. Data.gov. Accessed Nov 22, 2012, http://www.data.gov.

\section{AUTHOR BIOGRAPHIES}

HAZHIR RAHMANDAD is an Associate Professor in the Grado Department of Industrial and Systems Engineering at Virginia Tech. He received his Ph.D. in management from the Massachusetts Institute of Technology and his undergraduate degree is in Industrial Engineering from Sharif University of Technology, Tehran. Before joining the faculty of ISE, he was a post-doctoral associate at the MIT. He is an expert in System Dynamics modeling methodology and uses simulation models to build dynamic theories of organizational phenomena using a mixture of quantitative and qualitative data. Moreover, he is conducting research on comparing different simulation methodologies in the context of policy design for fighting and preventing epidemics and other public health challenges. His email address is hazhir@vt.edu and his web page is http://www.sdl.ise.vt.edu.

MOHAMMAD S. JALALI is a Ph.D. student in Management Systems Engineering in the Grado Department of Industrial \& Systems Engineering. He works with Hazhir Rahmandad at Virginia Tech's System Dynamics Lab. Mohammad is interested in simulation and model estimation methodologies and dynamic modeling for complex health care and social problems. Before joining the System Dynamics Lab, 
he was a researcher at the WV Industrial Assessment Center associated with the U.S. Department of Energy. His email address ismj@vt.edu and his web page is http://filebox.vt.edu/users/jalali.

HAMED GHODDUSI is a Postdoctoral Associate of Engineering Systems Division (ESD) at the Massachusetts Institute of Technology. He received his Ph.D. in 2011 from Vienna Graduate School of Finance (VGSF). Hamed's current research is mainly focused on Macro/Finance, Asset Pricing, and Energy and Commodity Finance. His email address is ghoddusi@mit.edu and his web page is

http://ghoddusi.com/ENE_webpage.html. 